\title{
Managing Temporal Dynamics of Filter Bubbles
}

\author{
Stefan Hirschmeier \\ University of Cologne \\ hirschmeier@wim.uni-koeln.de
}

\begin{abstract}
Filter bubbles have attracted much attention in recent years in terms of their impact on society. Whereas it is commonly agreed that filter bubbles should be managed, the question is still how. We draw a picture of filter bubbles as dynamic, slowly changing constructs that underlie temporal dynamics and that are constantly influenced by both machine and human. Anchored in a research setting with a major public broadcaster, we follow a design science approach on how to design the temporal dynamics in filter bubbles and how to design users' influence over time. We qualitatively evaluate our approach with a smartphone app for personalized radio and found that the adjustability of filter bubbles leads to a better cocreation of information flows between information broadcaster and listener.
\end{abstract}

\section{Introduction}

The internet becomes more and more personalized. A considerable portion of news, search engine results, and advertisements are already tailored to the interests of the users, and music and video streaming services use recommender systems to personalize their offerings. While recommender systems help users to overcome information overload [1], critics say that they tend to create filter bubbles [2] and lead to an unbalanced consumption and biased perspectives, often without users being aware of it, and have damaging potential to society [3-5].

Personalization and filter bubbles have attracted much attention in the last years in social science (e.g., [6-9]), computer science (e.g., [10, 11]), information systems (e.g., [12]), and law (e.g., [13, 14]) from technical, regulatory and societal viewpoints, and are also the subject of controversy (e.g., [15]). There is a large body of work emphasizing the importance of filter bubbles in opinion formation and social processes, and various approaches have been proposed how to make users aware of unbalanced content consumption (e.g., $[4,5,16])$ or how to avoid filter bubbles (e.g., [17]). However, the number of approaches proposed to managing filter bubbles is relatively small in comparison to the attention filter bubbles receive in terms of impact, and little design knowledge is known about how to make filter bubbles manageable for the user.

In order to effectively manage filter bubbles, it seems helpful to reconsider the nature of filter bubbles. Pariser, who coined the term filter bubble, described it as a state where non-transparent algorithms create a "unique universe of information" [2, p. 10] and isolate users from a diversity of viewpoints and content.

Regarding filter bubbles in a static way may suffice to explain the phenomenon of filter bubbles and their impact. But a static perspective does not yet provide insights into how to manage filter bubbles. As filter bubbles generally have a rather negative connotation, the wish to make them "burst" $([9,18])$ is obvious. But filter bubbles do not pop like soap bubbles or speculative bubbles. For example, in digital journalism, it is hard to find a single event like a new article with a different opinion, a new topic or a new insight that could serve as the needle to make the bubble burst. Approaches that deal with "bursting the filter bubble" (e.g., [4]) usually rather weaken the effect of the filter bubble and increase diverse exposure. The image of "bursting" the bubble is, therefore, somehow misleading. Even though the idea of bursting the bubble implicitly contains the beginning of a dynamic view and the management of filter bubbles, we need to understand filter bubbles as dynamic constructs and their manageability more thoroughly.

As long as filters are present, also filter bubbles can be present. As filter bubbles emerge from the application of a filter and lead to a preselection of content, and the filter is continually fed with new data from user feedback, filter bubbles tend to change over time, like a constant companion, sometimes weaker, sometimes stronger. The phenomenon that data changes over time and influences recommendations has been discussed as temporal dynamics in recommender systems literature [19-24]. We argue that also filter bubbles should be regarded as dynamic constructs that underlie temporal dynamics. In this view, filter bubbles may slowly inflate or deflate over time, according to the data the filter uses. 
There are different ways of dealing with filter bubbles. One approach is preventive - trying to avoid filter bubbles by running algorithms in the background to ensure balance and diversity (e.g., [17]). The other approach is permissive - allowing filter bubbles but giving the user control over them, allowing the user to play the corrective factor (e.g., $[4,5,16,25,26])$, and enabling a kind of value co-creation between the user and the recommender system. We want to focus on the latter - the manageability of filter bubbles.

As we regard filter bubbles as dynamic, technologydriven constructs, the question of managing filter bubbles is synonymous with the question of designing the dynamic change of filter bubbles. In most recommender systems applications, users can give likes and ratings for items and readjust them ex-post. But given dozens of likes every day, readjusting likes on an item-by-item basis can be quite time-consuming. Rather, adjustments (=temporal changes) to the user's interests (=likes) that make up the filter and subsequently the filter bubble call for a more efficient, co-creative way, allowing both the user and the system to change the user's filter bubble. Hence, we ask the question: How to design the change of filter bubbles over time and give users control over their dynamics?

\section{Related work}

The concept of filter bubbles is not without controversy. While the presence of filter bubbles as immaterial constructs emerging from algorithms that define a preselection of content has been widely accepted, critics say that there is little to no evidence, neither for filter bubbles nor for the related concept of echo chambers [15]. Both filter bubbles and echo chambers can lead to the phenomenon of a biased perception, but with different mechanisms. While filter bubbles have a purely algorithmic background, echo chambers can be seen as a social counterpart to filter bubbles and are created by like-minded people of a recipient who have similar opinions and thus lead to a reinforcement of the recipient's opinion [27, 28].

In the following, we present related work from two different fields. First, we refer to approaches that directly aim at avoiding and controlling filter bubbles, and second, we refer to value co-creation with regard to journalism, as giving users control over filter bubbles is a matter of co-creation of value between information provider and information recipient.

\subsection{Approaches to control filter bubbles}

Several approaches exist to avoid or reduce the risk of filter bubbles. Ekstrand et al. [29] propose a way to prevent filter bubbles by giving the user control over the algorithmic settings. This can be the selection of algorithms, or the possibility to combine one algorithm with another. They also propose to give the user control over the degree of diversity of the information from the user profile using adjustable variables [29]. Interactive Recommender Systems have been proposed that aim to give the user more control over the recommendation process and to improve transparency, e.g., [30].

Iaquinta et al. [31] present a concept of a hybrid recommender system that combines a content-based approach with serendipitous heuristics in order to avoid filter bubbles with surprising suggestions. Required for their serendipity method is a content-based recommender system, that implements their anomalies and exceptions approach. They follow the idea that content should not be recommended if it is too similar to items the user has already consumed.

Webster \& Vassileva [32] proposed an interactive visualization approach to explain and modify a collaborative filtering recommender system. The system shows the user which other users are most similar in terms of their preferences. Additionally, the user can change the degree of influence that the other users have on the recommendations individually for each user.

Nagulendra and Vassileva [25, 26] introduced an interactive visualization approach to illustrate and increase the awareness of the filter bubble in Online Social Networks. They designed their visualization based on a bubble metaphor to improve the comprehensibility of the filtering process in a way that is intuitive for the user. Category bubbles within the filter bubble show which categories are relevant from the recommender's perspective. The larger the bubble the greater the relevance of the category. Their system also offers the possibility to show which friends are most similar in terms of their interests. Additionally, users can modify the filter bubble by adding or deleting categories using a drag and drop function.

Furthermore, Bozdag and van den Hoven [5], Munson and Resnick [16], and Resnick et al. [4] present approaches on how to "burst the filter bubble" by making the user aware of unbalanced consumption.

\subsection{Value co-creation in journalism}

Value co-creation is one of the key axioms of service-dominant logic (SDL). As proposed by Vargo and Lusch [33], service rather than goods is the basis of economic exchange. Service is defined as "the application of specialized competences (knowledge and skills) through deeds, processes, and performances for the benefit of another entity or the entity itself' [33, p. 22]. SDL argues that value is (a) not created by a manufacturer or provider, (b) not embedded into 
products upon production, and (c) not exchanged in economic transactions (i.e., value in exchange). Rather, SDL argues that value is co-created when providers and customers interact - that is, when customers integrate their competencies with providers' service offerings (i.e., value in use or value in context), thereby making customers co-producers [33].

Transferred to traditional journalism, value is mainly created by the information provider (broadcasters, media houses, publishers) and consumed by the information recipient. The recipient has a rather passive role with hardly any opportunity for interaction.

In digital journalism, however, feedback channels exist that enable stronger value co-creation between information provider and recipient. As a result, the offering usually resembles an individual service offering rather than a mass offering.

Filter bubbles are mostly seen as intangible constructs with negative connotations that emerge from algorithms. However, under the value co-creation paradigm, allowing the user to modify the filter bubble may open up another facet of co-creating value between information provider and recipient.

\section{Method and Research Setting}

In order to achieve our research goal, we follow a design-oriented approach in the methodology of Design Science Research (DSR) [34-37]. Design science is a well-known research approach in IS and has been reemphasized in the last years. At the core of design science is the iterative design with continuous reflection and incremental refinement. So far, we conducted one design cycle, and aim at contributing a level 1 design science contribution in the terminology of Gregor and Hevner [38].

Our research took place in cooperation with a major public radio broadcaster in Germany. Public broadcasters have a public-service remit to fulfill, so the management of filter bubbles is of special interest to them. The research on the management of filter bubbles was embedded in a 2.5-year design-oriented research project on recommender systems in public broadcasting. In this research project, we built a smartphone app that contains an interactive radio player with a radio recommender system in the backend and a connection to the media library of the broadcaster. Next to the interactive radio player, the management of the user's filter bubble ${ }^{1}$ was also a feature of the smartphone app. It is important to note that the paper at hand focuses on the user's control over filter bubbles, not on the automatic prevention of filter bubbles.

\footnotetext{
${ }^{1}$ Actually, we do not manage the filter bubble itself, but the user-item-rating in the user profile that leads to the filter
}

Another focus of the research setting is the view on filter bubbles from a content perspective. The term filter bubble may describe both unbalanced topics and unbalanced opinions. Both are considered critical in literature: Information flows which, for example, almost completely omit political topics but mainly report on sports are just as critical as information flows which contain media bias in the form of mainly right- or leftoriented opinions. In our research setting, we focus on content filter bubbles, i.e., we look at filter bubbles from the perspective of content. Thus, we do not address opinions inside the filter bubble.

From a technical point of view, we design the mechanisms for temporal dynamics of filter bubbles and the corresponding recommender system.

\section{Design Approach}

In our design approach, we distinguish the terms items from topics. Items are articles recommended by the recommender system (e.g., with the title "Methane emissions reach new record level"), whereas topics represent aggregated areas of interest (e.g., "climate change"). Both may represent the interests of the user. Recommender systems always work on an item level (with user-item-ratings), whereas humans tend to think in topics.

\subsection{Design Requirements}

Through several workshops and discussions with station experts, we identified four design requirements (DR) for content filter bubble design. Below, we describe the design requirements we identified, accompanied by a rationale indicating which experts' statements led to the respective requirement.

DR1. Consumable. Filter bubble visualizations should represent the data used for filtering in an understandable and consumable way.

Rationale: Radio experts did not want to overwhelm listeners with information. Titles of radio pieces are rather long, and lists of liked or disliked titles cause even more data. Spoken in words of information quality dimensions [39], "appropriate amount of data" and "ease of understanding" would come close to DR1.

DR2. Accurate. Filter bubbles should reflect the data used for filtering in an accurate way.

Rationale: In the past, radio experts had categorized their content for a smartphone app, but found that accuracy can be an issue when merging data. Also,

bubble. However, for illustration purposes, we stick to the idea of managing the filter bubble. 
experts assume that listeners of public radio might have higher requirements for accuracy than others. Along with "ease of understanding" and "appropriate amount of data", "accuracy" is a typical information quality dimension [39].

DR3. Controllable. Users should be able to correct the bubble by removing and adding topics.

Rationale: This was the key requirement mentioned with regard to the public-service remit. The ability to control and manage the filter bubble was perceived as a new facet of providing public value. More control over a recommender system has shown a better user experience [40] and increased transparency and trust [30].

DR4. Expirable. Filter Bubbles should reflect the interests of the user with correct time reference.

Rationale: In previous prototypes (which did not include filter bubble management yet but only a personalized radio player), we found that test users were sensitive to outdated content. Just like content itself, also areas of interest may outdate, both of which are the reason for temporal dynamics effects in recommender systems. Together with radio experts, we concluded that filter bubbles should have a "drain", i.e., old topics should automatically lose in importance over time (if they are not renewed by new likes), so new topics can gain weight. Spoken in words of information quality dimensions [39], "timeliness" refers to DR4.

\subsection{Design Principles}

In the following, we present the design principles we developed to match the design requirements. For each design principle, we present a rationale that provides a connection to the design requirements and the respective challenges. Figure 1 provides an overview of how design requirements relate to design principles.

DP1. Interaction via word clouds. Word clouds with topics are a suitable way to represent filter bubbles on the presentation and interaction layer.

Rationale: While searching for a suitable visualization to match DR1, we tested several approaches and performed a test where we showed mockups to test users with topics in the form of lists, word clouds and a hybrid visualization, with the word clouds being preferred. Word clouds have also proven to be a suitable form for explaining recommendations in literature [41-43]. Because of their cloud shape, word clouds are much better at representing a bubble metaphor than other visualizations such as lists. The interaction via word clouds allows humans to interact in an intuitive way with the filter bubble, as humans think in topics, whereas machines operate on a more detailed level with user-item-ratings. Therefore, DP1 matches DR1 (see Figure 1).

Challenges: So far, word clouds have mostly been used to create static images and are rather unknown for being editable.

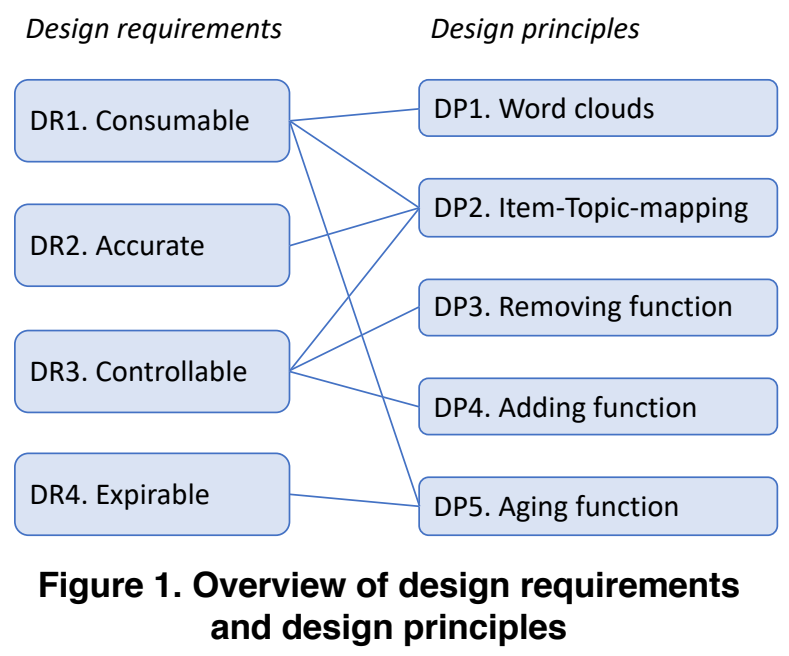

DP2. Item-Topic-Mapping. An item-topic-mapping should be used to provide data for the word cloud.

Rationale: The human-consumable presentation described in DP1 requires the supply of corresponding data. DP2 is therefore the fundament to DP1. To covert data from user-item-ratings into a human-consumable form, metadata must be generated to enable an itemtopic-mapping.

Challenges: Different methods exist to generate metadata: (a) manually by archivists, editors, or users, or (b) automatically by natural language processing (NLP) techniques such as named entity recognition [44], part-of-speech-tagging (PoS) [45], term frequencies, supervised and unsupervised machine learning (e.g., [46, 47]), or transfer learning approaches (e.g., [48, 49]). In practice, manual tagging of keywords is often not economically feasible, or not to the required extent, so often only part of the media content is tagged by human agents. We chose a simple approach of automatic keyword generation by using the term frequency-inverse document frequency method ( $\mathrm{tf}-\mathrm{idf}$ ) $[46,50]$ on the teaser texts and selecting the nouns only. More sophisticated approaches in NLP are imaginable. The topics are associated with items in a 1:n way, i.e., several topics can be tagged to one item. DP2 serves to fulfill DR1, DR2, and DR3 (see Figure 1).

DP3. Removing function. A function to manually remove topics should be implemented.

Rationale: According to DR3, users should be able to manually remove topics from their word cloud (filter bubble), e.g., by dragging and dropping topics from the 
inside of the bubble to the outside. When a topic is removed by the user, we identify all items related to that topic using the item-topic-mapping described in DP2 and reset all likes of the identified items.

Challenges: As we have a 1:n relationship of items to topics, we unintentionally also decrease other topics in their importance. For example, an item that the user liked is associated with the topics "Corona" and "health". Assume the user profile consists of various topics with different weights (number of likes shown in brackets), among them "Corona" (1), "health" (9), and "robots" (6). If the user manually removes the topic "Corona" from the filter bubble, also the topic "health" would lose importance, resulting in a user profile of "Corona" (0), "health" (8), and "robots" (6). However, the more data the user profile contains, the more detailed the user profile is and the less likely co-occurrence and dependencies on topics are.

DP4. Adding function. A function to manually add topics should be implemented.

Rationale: According to DR3, users should also be able to add topics. Using the item-topic-mapping described in DP2, we can identify the items related to the new manually added topics.

Challenges: We do not have a symmetric situation to removing topics (as described in DP3). When deleting topics, the user is aware that his former likes are likely to be reset. When adding topics, in contrast, the user will most likely not expect that some item-likes are set automatically. So, when a user adds topics to his filter bubble, we decided that items concerning that topic should not be liked automatically but queued by the recommender system for upcoming recommendations. This way, by adding topics the user will not immediately influence the recommender system but time-delayed.

DP5. Aging function. Older ratings should decrease in importance.

Rationale: DP5 is directly derived from DR4 in order to represent the time reference of user interests. What was of interest last year is not necessarily of interest today.

Challenges: The tricky part is that the aging function should be congruent for both a) the database for the recommender system and $b$ ) the visualization for the user. Regarding b), topics that lose in importance can also be displayed less important (e.g., by smaller fonts) and regarding a) the same aging function has to be applied in the recommendation engine, otherwise, the word cloud would fail to provide an accurate visualization (as required by DR1). The simplest way of an aging function is to give an expiry date to user-itemratings, so no "smooth fade-out" function has to be implemented. Therefore, DP5 serves DR4 in conjunction with DR1.

\subsection{Designing the data flow in filter bubbles}

Figure 2 provides a view on the dynamic data flow in filter bubbles as designed in our approach. The filter bubble is visualized by a word cloud with different topics as depicted in DP1. For better understanding, the design principles are also allocated in Figure 2.

4.3.1. Sources for the filter bubble. The filter bubble has two sources. The first source is the likes and ratings from the user that are collected by the recommender system (upper left corner in Figure 2). In order to visualize the topics associated with these rated items, the item-topic-mapping is required (DP2). The second source is the manual adding of topics (lower-left corner in Figure 2) as described in DP4.

4.3.2. Drains for the filter bubble. The filter bubble has two drains. The first drain is an automatic aging of user-item-ratings (upper right corner in Figure 2) as described in DP5. The second drain is the manual removing of topics (lower right corner in Figure 2) as described in DP 3.

4.3.3. Co-creating the bubble data. Over time, recommender systems collect feedback data from the user and build up a user profile in the form of user-itemratings. Depending on the implementation, explicit and/or implicit feedback is collected. As a result, the recommender system builds up a foreign image of the user, which is not necessarily in line with the self-image [51] of the user.

Research has shown that users might hesitate to feed recommender systems with feedback when they know that the feedback is stored in their user profile and they lose control over the data [52]. Algorithms have a hard time to distinguish short-term from long-term interests [53] and which characteristic of an item is interesting for a user in particular. In other words, feedback possibilities of recommender systems are well-suited for data collection, but less suited for adjusting and managing, and users have hardly any control over the data once it is inside the system.

The explicit or implicit feedback that users give to recommended items can already be seen as a form of value co-creation [54]. The user actively or passively discloses information that is of use to render information more individually towards him/her. Value is not created by the information provider alone, but in co-creation with the recipient, resulting in personalized information streams. 


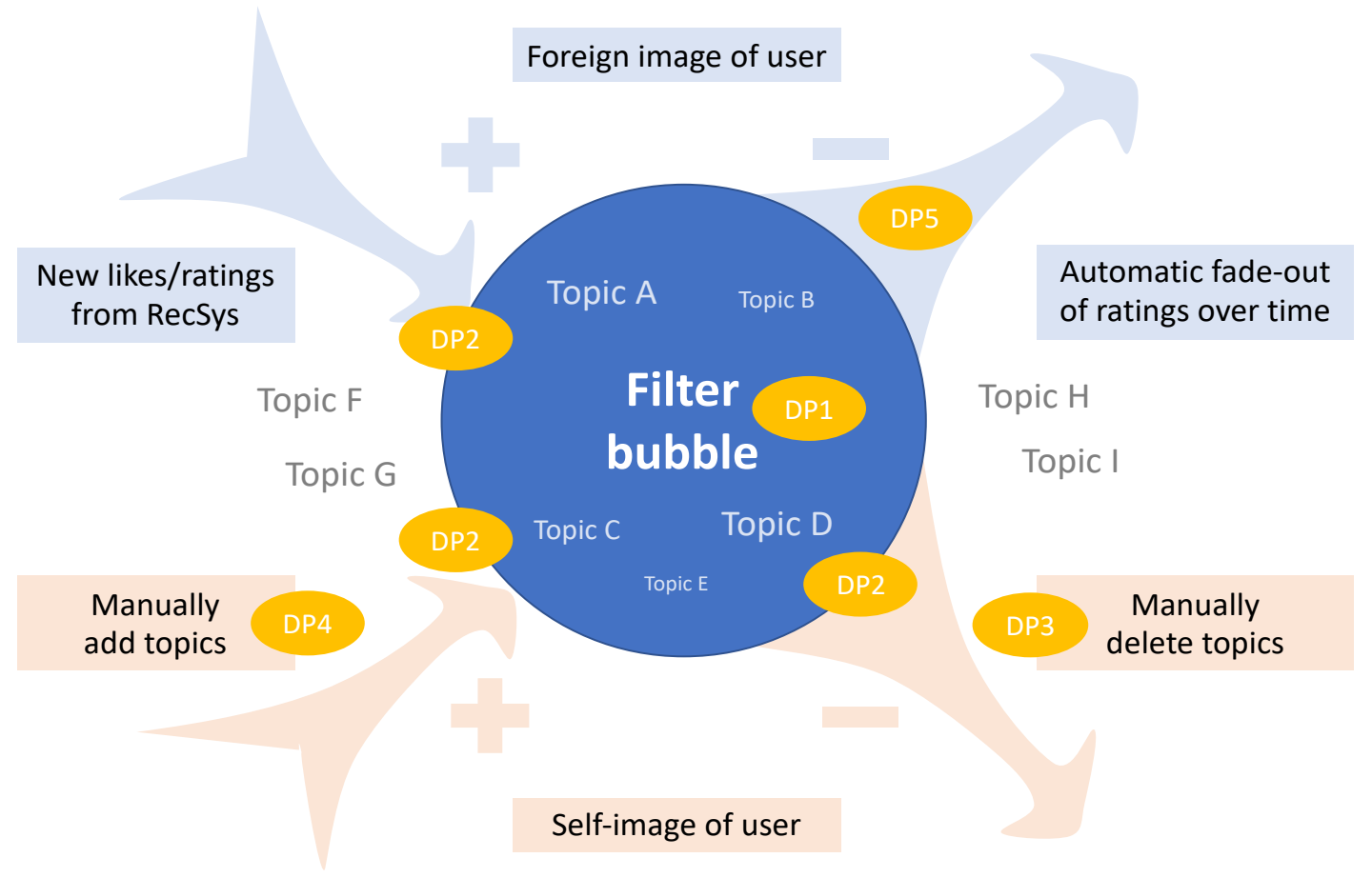

Figure 2. Temporal dynamics of a filter bubble

With our approach, we enhance the concept of value co-creation. We allow users to correct the foreign image that the filter algorithm has of them with their selfimage. The filter bubble as designed in our approach can be used to continuously adjust foreign image and selfimage. Also, users can undo likes or views (as an implicit expression of interest) that were only of temporary interest for the user. In comparison, the possibility to give likes and dislikes provides only limited control over the data stored in the user profile [55] and the resulting filter bubble.

4.3.4. Deflating and inflating bubbles. As a matter of continuous adjustment, the temporal dynamics between two sources and two drains of the bubble lead to temporary deflations and inflations of the bubble.

The smaller the filter bubble get (few topics inside), the stronger it personalizes. The bigger the bubble gets (many topics inside), the more diverse personalization becomes. When a user manually removes topics from inside the filter bubble (or drags them out of the bubble), diversity decreases (all else being equal). When a user manually adds topics (or drags them from outside the bubble into the bubble) diversity increases (ceteris paribus). Speaking in metaphors again, filter bubbles do not burst from getting too big like balloons do. They can expand infinitely, and their effect disappears the greater they become.

\section{Evaluation}

\subsection{Evaluation plan}

For evaluation purposes, we developed a web app for smartphones that included three screens (Figure 3), (a) a simple player (play, pause, skip, rewind 15 seconds) with recommender feedback interactions, (b) a screen with a history of all consumed items and the user's rating in list form, and (c) visualization of the users' filter bubble in which users could also manage their filter bubbles. The prototype was connected to the media library of a public broadcaster. In the history screen, a list of all consumed items was shown and the corresponding rating. Users were able to change the ratings in the list ex-post and to reset all ratings. In the filter bubble screen, users could explore topics in their filter bubble, remove topics from their filter bubble or reset the whole bubble. The color coding and orientation of words were random. In this prototype, users were however not yet able to add topics.

Considering the early stage of developing filter bubble management for digital journalism, we chose a qualitative evaluation design with personal interviews. We developed an interview guideline for descriptive face-to-face interviews to get a rich description of the perception and acceptance of filter bubble management. 
During the prototype development, we pre-tested our prototype with ten test listeners to see if there are major flaws we need to adjust in the prototype or our interview guideline. After that, for the subsequent evaluation, we conducted 34 interviews of 10 to 15 minutes between November 2019 and January 2020. As we decided to gather feedback from both regular radio listeners and non-listeners, we interviewed $50 \%$ listeners and $50 \%$ non-listeners. The age of our test persons ranged from 21 to 72 years.

After a brief introduction to our research project, we presented the prototype to the participants and asked them to express any experience and thought following the think-aloud method [56]. We did not explain the features of the prototype but wanted to see how users get along themselves, how intuitive the approach is, and hear what participants think while exploring the prototype. We helped when participants missed features or had problems using the prototype. We asked participants to screen at least 15 content items and like a couple of them to populate the filter bubble. After participants had used all features and produced enough data, we asked them in which regard they prefer to use the history (list of items) and in which regard they prefer to use the filter bubble to adjust their likes and interests.
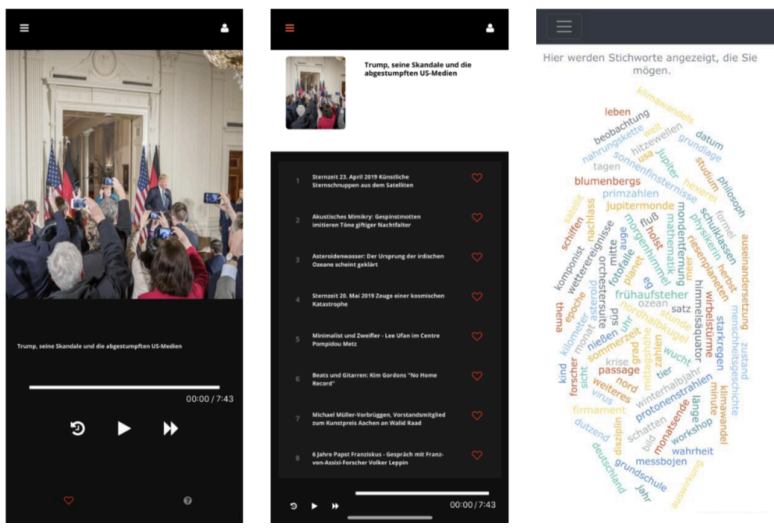

Figure 3. Prototype with player (left), history (middle) and filter bubble visualization (right)

\subsection{Evaluation results}

5.2.1. Perception of the word cloud in general. Some participants intuitively perceived value in seeing the filter bubble and being able to adjust it. "This is a great feature. I would use this word cloud to train the recommender system. In the first weeks, I would probably check regularly, and afterwards only occasionally." Next to the idea of training the recommender system after startup, other participants connected to issues they had with recommender systems before, such as, „on other platforms like Spotify or
Youtube I sometimes wonder how my feed comes about. Especially on Youtube, I am annoyed by this. I would even tend not to click on certain things because I know that it will flush videos from certain areas into the feed that I don't want to see."

Most of the participants appreciated further interaction possibilities with the recommender system, and regarding the adjustability and the visualization of the filter bubble using the word cloud, users largely agreed that the ability to inspect the filter bubble, to adjust specific keywords and to reset the entire profile is very meaningful and valuable, from which we conclude a positive evaluation of DP1. Our findings are in line with [41], who found word clouds to be a suitable form for visualization of recommendation results.

5.2.2. Perception of topics inside the word cloud. The topics displayed by the filter bubble caused much attention. Some topics were as expected by participants, other topics were not expected and made participants curious. So, the evaluation of DP2 (the item-topicmapping), on the one hand, resulted in acceptance (expected topics), and on the other hand resulted in surprise (unexpected topics), which led to a greater interest in scrutinizing and adjusting the filter bubble.

Often, it was not intuitive to participants that the words are editable, so we had to explicitly mention that words can be removed. However, after removing words from the bubble, all participants spoke positively about the removing function (DP3).

However, while most participants generally agreed with a word cloud visualization, participants also criticized the way we implemented the visualization. Some participants were irritated by the orientation of words, the color code, and complained about the chaotical order, such as, "I always have to turn the phone to read the words", and "some words are blue, some are yellow, and I don't know why". Those participants perceived the visualization of the history (list of items) as more pleasant and less confusing in direct comparison. Also, participants said they were used to working with lists, whereas the handling of interactive word clouds was rather unfamiliar to them. As a consequence of the chosen evaluation design, we could not separate functionality from the design of features, and we had not optimized usability. Other visualizations of the word cloud, such as different color codes, fonts, font sizes, text orientation and local arrangements such as clusters of words might result in a better acceptance of the word cloud and better intuitiveness.

We were not able to evaluate DP4 and DP5 as the adding function was not yet implemented in this design cycle (but we expect the user feedback not so much different from the topic removing function) and the 
aging function needs a different long-term evaluation setup.

\section{Discussion and limitations}

In this research, we proposed an approach to manage filter bubbles for digital journalism by conceptualizing and designing the temporal dynamics of filter bubbles. We allowed users to interact with the filter bubble for a continuous value co-creation to align foreign image and self-image of the user. While this research provides a conceptual basis for the management of filter bubbles at an early stage, it can already inform providers of digital journalism how to design filter bubbles from a dynamic perspective and allow users to better co-create value by enhanced interaction possibilities.

Contribution to science. We contributed a level 1 design science contribution in the terminology of Gregor and Hevner [38] that may support nascent design theories for the management of filter bubbles.

Contribution to practice. The results from this research might not only be of valuable insight for public broadcasters that have a public-service remit to fulfil. Manageable filter bubbles might also be of value to private media offers in journalism, as they may increase trust in recommender systems and have the potential to strengthen customer loyalty.

Limitations. Surely, our approach is not without limitations. With the word cloud and the item-topicmapping, we assume that the user liked the content of the item (i.e., article). However, we cannot be sure if the user liked the content, it could have also been the author, the format, the writing style, or something else. It is also possible that the user only liked part of the item. We do not know exactly - but recommender systems do neither. Still, we have an additional bias in the itemtopic-mapping, and, in the worst case, we might present an image to the user that does not truly reflect his/her user-item-ratings, and therefore not truly fulfill DR2 (accuracy).

Also, there is a possible contradiction in the design requirements. Consumability (DR1) and accuracy (DR2) do not always go hand in hand, as consumability might require a reduction of information and a loss of accuracy. A possible trade-off between consumability and accuracy can therefore be seen as a limitation in the requirements. Nevertheless, the topic-item-mapping seems to be an motivator and enabler for interaction with the filter bubble.

Future Research. Future research should analyze how to improve the usability of interactive filter bubbles concerning color coding, font, font size, orientation, and local arrangements. Also, the level of granularity of topics may be important for research (e.g., what to display when a user is interested in football, but not in tennis) with a taxonomy in the background.

In this research, we focused on content filter bubbles. As a next step, also an opinion perspective (political orientation) could be integrated into the filter bubble, raising the question of how to categorize opinions, how to integrate them into the bubble visualization, and how to adjust political orientation. Furthermore, the bubble we designed represents the positive interests of the user, whereas topics outside the bubble can still be part of the recommendation, as the user is still open to those topics, but not intensely interested. However, there might be topics that users want to avoid. While this may be important for public service journalism, it might also be of interest to private media houses to express and adjust negative interests. Further research has to investigate the solution corridor of manageable filter bubbles for digital journalism and their temporal dynamics.

\section{Acknowledgments}

This work was supported by Deutschlandradio in a joint research project with the University of Cologne. Many thanks to Jan Janka, Markus Waldhauser and Dr. Nicola Balkenhol for their support.

\section{References}

[1] F. R. Ricci Lior; Shapira, Bracha, "Introduction to Recommender Systems Handbook," in Recommender Systems Handbook, F. R. Ricci Lior; Shapira, Bracha; Kantor, Paul B., Ed. Boston, MA, USA: Springer, 2011, pp. 1-30.

[2] E. Pariser, The Filter Bubble: What The Internet Is Hiding From You. Penguin UK, 2011.

[3] N. Pöchhacker, M. Burkhardt, A. Geipel, and J.-H. Passoth, "Interventionen in die Produktion algorithmischer Öffentlichkeiten: Recommender Systeme als Herausforderung für öffentlich-rechtliche Sendeanstalten,"kommunikation@gesellschaft, vol. 18, 2017.

[4] P. Resnick, R. K. Garrett, T. Kriplean, S. A. Munson, and N. J. Stroud, "Bursting your (filter) bubble: strategies for promoting diverse exposure," 2013, pp. 95-100. doi: 10.1145/2441955.2441981.

[5] E. Bozdag and J. van den Hoven, "Breaking the filter bubble: democracy and design," Ethics Inf Technol, vol. 17, no. 4, pp. 249-265, Dec. 2015, doi: 10.1007/s10676-015-9380-y.

[6] Q. V. Liao and W. T. Fu, "Beyond the filter bubble: Interactive effects of perceived threat and topic involvement on selective exposure to information," presented at the 31st Annual CHI Conference on Human Factors in Computing Systems: Changing Perspectives, CHI 2013, 2013. doi: $10.1145 / 2470654.2481326$. 
[7] D. Nikolov, D. F. M. Oliveira, A. Flammini, and F. Menczer, "Measuring online social bubbles," PeerJ Computer Science, vol. 1, p. e38, Dec. 2015, doi: 10.7717/peerj-cs.38.

[8] C. Matt, A. Benlian, T. Hess, and C. Weiß, "Escaping from the Filter Bubble? The Effects of Novelty and Serendipity on Users' Evaluations of Online Recommendations," Dec. 2014.

[9] M. Haim, A. Graefe, and H.-B. Brosius, "Burst of the Filter Bubble?," Digital Journalism, pp. 330-343, Jul. 2018.

[10] M. Taramigkou, E. Bothos, K. Christidis, D. Apostolou, and G. Mentzas, "Escape the bubble: guided exploration of music preferences for serendipity and novelty," in Proceedings of the 7th ACM conference on Recommender systems, Hong Kong, China, Oct. 2013, pp. 335-338. doi: 10.1145/2507157.2507223.

[11] P. Symeonidis, L. Coba, and M. Zanker, "Counteracting the filter bubble in recommender systems: Novelty-aware matrix factorization," Intelligenza Artificiale, vol. 13, no. 1, pp. 37-47, Jan. 2019, doi: 10.3233/IA-190017.

[12] T. T. Nguyen, P.-M. Hui, F. M. Harper, L. Terveen, and J. A. Konstan, "Exploring the filter bubble: the effect of using recommender systems on content diversity," 2014, pp. 677-686. doi: $10.1145 / 2566486.2568012$.

[13] F. J. Zuiderveen Borgesius, D. Trilling, J. Möller, B. Bodó, C. H. de Vreese, and N. Helberger, "Should we worry about filter bubbles?," Internet Policy Review, vol. 5, no. 1, Mar. 2016, doi: 10.14763/2016.1.401.

[14] N. Helberger, "On the Democratic Role of News Recommenders," Digital Journalism, vol. 7, no. 8, pp. 993-1012, Sep. 2019, doi: 10.1080/21670811.2019.1623700.

[15] A. Bruns, "Filter bubble," Internet Policy Review, vol. 8, no. 4, Nov. 2019, Accessed: Aug. 30, 2021. [Online]. Available: https://policyreview.info/concepts/filter-bubble

[16] S. Munson and P. Resnick, "Encouraging Reading of Diverse Political Viewpoints with a Browser Widget," presented at the Proceedings of the 7th International Conference on Weblogs and Social Media, ICWSM, 2013.

[17] S. Hirschmeier and V. Beule, "Compliance of Personalized Radio with Public-Service Remits," Singapore, 2018. doi: 10.1145/3213586.3226204.

[18] A. Abisheva, D. Garcia, and F. Schweitzer, "When the filter bubble bursts: collective evaluation dynamics in online communities," in Proceedings of the 8th ACM Conference on Web Science, New York, NY, USA, May 2016, pp. 307-308. doi: 10.1145/2908131.2908180.

[19] W. Hong, L. Li, and T. Li, "Product recommendation with temporal dynamics," Expert Systems with Applications, vol. 39, no. 16, pp. 12398-12406, Nov. 2012, doi: 10.1016/j.eswa.2012.04.082.

[20] Y. Koren, "Collaborative filtering with temporal dynamics," in Proceedings of the 15th ACM SIGKDD international conference on Knowledge discovery and data mining, Paris, France, Jun. 2009, pp. 447-456. doi: 10.1145/1557019.1557072.

[21] K. Ji, R. Sun, W. Shu, and X. Li, "Next-song recommendation with temporal dynamics," Knowledge-Based Systems, vol. 88, pp. 134-143, Nov. 2015, doi: 10.1016/j.knosys.2015.07.039.

[22] C. Rana and S. K. Jain, "A study of the dynamic features of recommender systems," Artificial Intelligence Review, vol. 43, no. 1, pp. 141-153, Jan. 2015, doi: 10.1007/s10462-012-9359-6.

[23] N. Koenigstein, G. Dror, and Y. Koren, "Yahoo! music recommendations: modeling music ratings with temporal dynamics and item taxonomy," in Proceedings of the fifth ACM conference on Recommender systems, New York, NY, USA, Oct. 2011, pp. 165-172. doi: 10.1145/2043932.2043964.

[24] J. L. Moore, S. Chen, D. Turnbull, and T. Joachims, "Taste Over Time: The Temporal Dynamics of User Preferences," 2013.

[25] S. Nagulendra and J. Vassileva, "Understanding and controlling the filter bubble through interactive visualization: a user study," in Proceedings of the 25th ACM conference on Hypertext and social media, Santiago, Chile, Sep. 2014, pp. 107-115. doi: $10.1145 / 2631775.2631811$

[26] S. Nagulendra and J. Vassileva, "Providing awareness, explanation and control of personalized filtering in a social networking site," Inf Syst Front, vol. 18, no. 1, pp. 145-158, 2016, doi: 10.1007/s10796-015-9577-y.

[27] S. Flaxman, S. Goel, and J. M. Rao, "Filter Bubbles, Echo Chambers, and Online News Consumption," Social Science Research Network, Rochester, NY, SSRN Scholarly Paper ID 2363701, Mar. 2016. Accessed: Apr. 06, 2018. [Online]. Available: https://papers.ssrn.com/abstract $=2363701$

[28] R. K. Garrett, "Echo chambers online?: Politically motivated selective exposure among Internet news users," Journal of Computer-Mediated Communication, vol. 14, no. 2, pp. 265-285, Jan. 2009, doi: 10.1111/j.1083-6101.2009.01440.x.

[29] M. D. Ekstrand, D. Kluver, F. M. Harper, and J. A. Konstan, Letting Users Choose Recommender Algorithms: An Experimental Study. Association for Computing Machinery, 2015. doi: https://doi.org/10.1145/2792838.2800195.

[30] C. di Sciascio, V. Sabol, and E. E. Veas, "Rank As You Go: User-Driven Exploration of Search Results," in Proceedings of the 21st International Conference on Intelligent User Interfaces, New York, NY, USA, 2016, pp. 118-129. doi: 10.1145/2856767.2856797.

[31] L. Iaquinta, M. d Gemmis, P. Lops, G. Semeraro, M. Filannino, and P. Molino, "Introducing Serendipity in a Content-Based Recommender System," in 2008 Eighth International Conference on Hybrid Intelligent Systems, Sep. 2008, pp. 168-173. doi: 10.1109/HIS.2008.25.

[32] A. Webster and J. Vassileva, "The keepup recommender system," in Proceedings of the 2007 ACM conference on Recommender systems, Minneapolis, MN, USA, Oct. 2007, pp. 173-176. doi: 10.1145/1297231.1297264. 
[33] S. L. Vargo and R. F. Lusch, "Evolving to a New Dominant Logic for Marketing," Journal of Marketing, vol. 68 , no. 1, pp. 1-17, Jan. 2004, doi: 10.1509/jmkg.68.1.1.24036.

[34] K. Peffers, T. Tuunanen, M. Rothenberger, and S. Chatterjee, "A Design Science Research Methodology for Information Systems Research," J. Manage. Inf. Syst., vol. 24, no. 3, pp. 45-77, Dec. 2007, doi: 10.2753/MIS0742-1222240302.

[35] A. Hevner, "A Three Cycle View of Design Science Research," Scandinavian Journal of Information Systems, vol. 19, 2007.

[36] J. Iivari, "Distinguishing and contrasting two strategies for design science research," Eur J Inf Syst, vol. 24, no. 1, pp. 107-115, Jan. 2015, doi: 10.1057/ejis.2013.35.

[37] V. K. Vaishnavi and W. Kuechler, Design Science Research Methods and Patterns: Innovating Information and Communication Technology. 2015.

[38] S. Gregor and A. R. Hevner, "Positioning and presenting design science research for maximum impact," vol. 37, no. 2, pp. 337-A336, 2013.

[39] R. Y. Wang and D. M. Strong, "Beyond Accuracy: What Data Quality Means to Data Consumers," Journal of Management Information Systems, vol. 12, no. 4, pp. 5-33, Apr. 1996, doi: 10.2307/40398176.

[40] B. P. Knijnenburg, M. C. Willemsen, Z. Gantner, H. Soncu, and C. Newell, "Explaining the user experience of recommender systems," User Modeling and UserAdapted Interaction, vol. 22, no. 4-5, pp. 441-504, Oct. 2012, doi: 10.1007/s11257-011-9118-4.

[41] C.-H. Tsai and P. Brusilovsky, "Providing Control and Transparency in a Social Recommender System for Academic Conferences," in Proceedings of the 25th Conference on User Modeling, Adaptation and Personalization - UMAP '17, Bratislava, Slovakia, 2017, pp. 313-317. doi: 10.1145/3079628.3079701.

[42] C.-H. Tsai and P. Brusilovsky, "Explaining recommendations in an interactive hybrid social recommender," in Proceedings of the 24th International Conference on Intelligent User Interfaces, Marina del Ray, California, Mar. 2019, pp. 391-396. doi: 10.1145/3301275.3302318.

[43] D. De Nart and C. Tasso, "A Personalized Conceptdriven Recommender System for Scientific Libraries," Procedia Computer Science, vol. 38, pp. 84-91, Jan. 2014, doi: 10.1016/j.procs.2014.10.015.

[44] O. Etzioni et al., "Unsupervised Named-entity Extraction from the Web: An Experimental Study," Artif. Intell., vol. 165, no. 1, pp. 91-134, Jun. 2005, doi: 10.1016/j.artint.2005.03.001.

[45] H. Schmid, "Probabilistic Part-of-Speech Tagging Using Decision Trees," in International Conference on
New Methods in Language Processing, Manchester, UK, 1994, pp. 44-49.

[46] A. Hulth, "Improved Automatic Keyword Extraction Given More Linguistic Knowledge," in Proceedings of the 2003 Conference on Empirical Methods in Natural Language Processing, Stroudsburg, PA, USA, 2003, pp. 216-223. doi: 10.3115/1119355.1119383.

[47] S. Hirschmeier, J. Melsbach, D. Schoder, and S. Stahlmann, "Improving Recall and Precision in Unsupervised Multi-Label Document Classification Tasks by Combining Word Embeddings with TF-IDF," ECIS 2020 Research Papers, 2020, [Online]. Available: https://aisel.aisnet.org/ecis2020_rp/99

[48] J. Devlin, M.-W. Chang, K. Lee, and K. Toutanova, "BERT: Pre-training of Deep Bidirectional Transformers for Language Understanding," 2019, pp. 4171-4186. doi: 10.18653/v1/N19-1423.

[49] Y. Liu et al., "RoBERTa: A Robustly Optimized BERT Pretraining Approach," arXiv:1907.11692 [cs], Jul. 2019, Accessed: Mar. 25, 2020. [Online]. Available: http://arxiv.org/abs/1907.11692

[50] R. Baeza-Yates and B. Ribeiro-Neto, Modern Information Retrieval. New York: Addison Wesley, 2010.

[51] J. A. Bailey, "Self-image, self-concept, and selfidentity revisited.," J Natl Med Assoc, vol. 95, no. 5, pp. 383-386, May 2003.

[52] I. Mohallick, K. De Moor, Ö. Özgöbek, and J. A. Gulla, "Towards new privacy regulations in europe: Users' privacy perception in recommender systems," 319-330, 2018, doi: 10.1007/978-3-030-05345-1_27.

[53] M. An, F. Wu, C. Wu, K. Zhang, Z. Liu, and X. Xie, "Neural News Recommendation with Long- and Shortterm User Representations," in Proceedings of the 57th Annual Meeting of the Association for Computational Linguistics, Florence, Italy, Jul. 2019, pp. 336-345. doi: 10.18653/v1/P19-1033.

[54] S. Hirschmeier, V. Beule, and R. Tilly, "Translating Editorial Work into Algorithms for Personalized Radio Streams," Journal of Radio \& Audio Media, vol. 0, no. 0, pp. 1-22, 2020, doi: 10.1080/19376529.2019.1704760

[55] B. Zhang, N. Wang, and H. Jin, "Privacy Concerns in Online Recommender Systems: Influences of Control and User Data Input," 2014, pp. 159-173. Accessed: Sep. 05, 2021. [Online]. Available:

https://www.usenix.org/conference/soups2014/proceedi ngs/presentation/zhang

[56] E. Charters, "The Use of Think-aloud Methods in Qualitative Research - An Introduction to Think-aloud Methods," Brock Education Journal, vol. 12, no. 2, Art. no. 2, Jul. 2003, doi: 10.26522/brocked.v12i2.38. 